\title{
SIMULATION FOR PERFORMANCE EVALUATION OF THE HOUSEKEEPING PROCESS
}

\author{
Pasquale Legato \\ Rina Mary Mazza \\ Roberto Trunfio \\ Department of Electronics, Computer and System Sciences, University of Calabria \\ Via P. Bucci 42C \\ 87036 Rende (CS), Italy
}

\begin{abstract}
The literature on the optimization of container terminal logistics has recently focused on space assignment and equipment management within the yard sub-system. Yet, to our knowledge, no models have been proposed for housekeeping, i.e. the process according to which a container is moved from one yard position to another during its stay in the terminal's storage area. Housekeeping has only been addressed as a conceptual choice lying behind yard operating rules when dealing with empirical investigations for intelligent yard stacking. Here we propose a queuing-based representation of the current housekeeping process in a real container terminal and solve it by discrete-event simulation to $i$ ) assess the efficiency of the housekeeping operations under unforeseen events or process disturbances and ii) estimate the related productivity and waiting phenomena which, in turn, affect the vessel turn-around time. Sample results returned by the simulator are presented to illustrate possible usage via scenario analysis.
\end{abstract}

\section{INTRODUCTION}

The consolidated importance of containerized traffic in global trade is evidenced by the growth of container volumes handled. According to Container Trades Statistics (Container Trades Statistics 2012), in the past two years Europe alone has accounted for a 16\% increase of imported and exported containers (from 32,725,900 in 2009 to 37,944,400 in 2011 measured in TEUs - twenty-foot equivalent units, a measure used for capacity in container transportation). As a result, container terminals are stressed to accommodate the growing container traffic, usually, within the same physical area dedicated to container storage, while providing for a more careful management of the facility equipment.

In this context, container handling throughout the major logistic sub-systems of the terminal becomes a key issue to maintain high levels of productivity and quality of service. Most of the logistic operations provided in a container terminal are carried out throughout the interaction of four sub-systems, as illustrated in Figure 1.

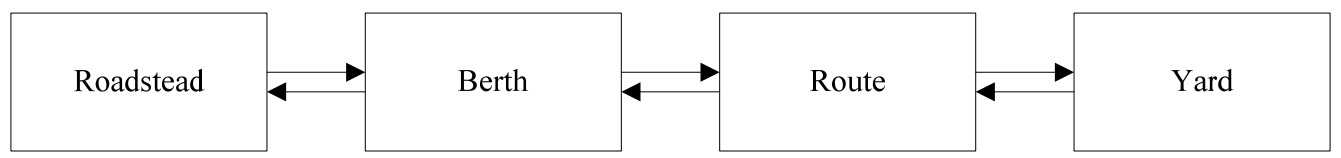

Figure 1: The main subsystems in a maritime container terminal

The premier form of vessel service occurs in the roadstead: the terminal operator monitors vessel tugging during arrivals via the entrance channel. Once in the port, vessels are moored along the berth, where cranes are assigned, deployed and scheduled in order for container discharge/loading operations to 
take place. During these operations, container transfer from the quay to the yard and vice versa occurs according to specific routes, while container stacking and retrieval is carried out on the terminal yard. As soon as discharge/loading is completed, vessels are unberthed and they leave the port via the exit channel.

With respect to the overall operation cycle described above, the time between vessel arrival and departure is known as vessel turn-around time. One of the major objectives of a maritime container terminal consists in minimizing the vessel turn-around time: obviously, the smaller the time, the greater the productivity. To do so, terminal operators can consider both on-line and off-line planning options.

On-line planning is likely to occur when information delivery is provided in real-time. It can either be based on the exploitation of a variable number of resources or the dynamic allocation of a fixed number of resources (for instance, using load balancing policies to roughly obtain the same workload and, thus, utilization for the available resources). On the other hand, off-line planning is usually pursued when detailed information is available before performing actual loading and discharge operations. In the present paper, to speed up vessel turn-around time we will consider off-line planning for both loading and discharge operations.

To begin with, loading operations can be sped up by prearranging the containers on the yard to mirror their final positions on the vessel. In other words, by converting the current yard layout into an ideal layout, no unproductive container moves or reshufflings on the yard will be necessary during actual loading operations. This concept is known as re-marshalling (Kim and Bae 1998; Lee and Hsu 2007; Hirashima 2009; Lee and Chao 2009; Yu , Cheng, and Ting 2009; Choon et al. 2010; Bortfeldt and Forster 2012; Huang and Lin 2012) and a simplified illustration is given in Figure 2.

As one may see, storage space in both terminal yard blocks and containerships is organized in bays, where the size of a bay is determined by the number of lines (rows) per the number of containers stacked in height (tiers). By re-marshalling and setting the proper direction of work (i.e. left to right and top to bottom on the yard bay and right to left and bottom to top on the vessel bay), no reshuffles, nor delays will occur for a given container loading pattern, which in the specific case in Figure 2 is represented by the order of the numbered containers.
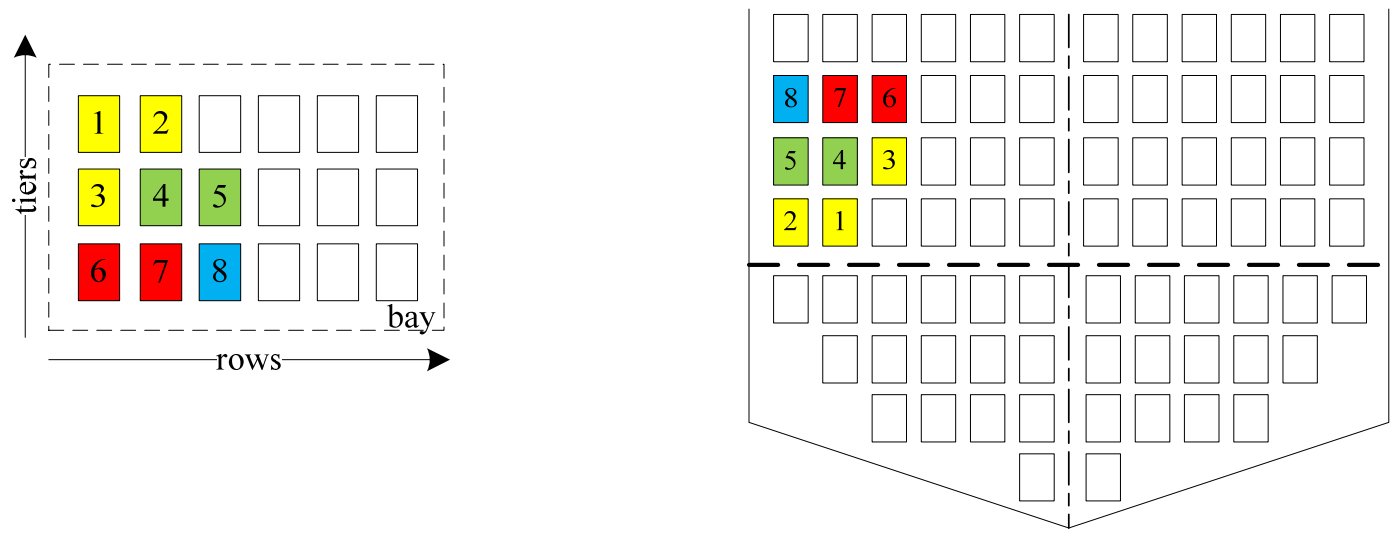

a) bay view (vertical section) of a yard block

b) bay view (vertical section) of a vessel

Figure 2: Example of how the bays of a yard block should mirror the bays of a vessel

As for speeding up operations in the discharge case, an off-line modus operandi can be employed when the distance between the vessel discharge point along the berth and the container stacking point on the yard is large. A practical option consists in performing an indirect discharge, i.e. temporarily leaving the containers in a buffer area and resuming container transfer to final positions on the yard later on. The buffer may be an independent common front area as the one illustrated in Figure 3, in which the distance to cover during discharge operations is marked with a continuous line, whereas the distance to cover afterwards is represented by a dotted line. When switching from situation a) to situation $b$ ) how and why time savings are achievable during actual operations is quite clear. 


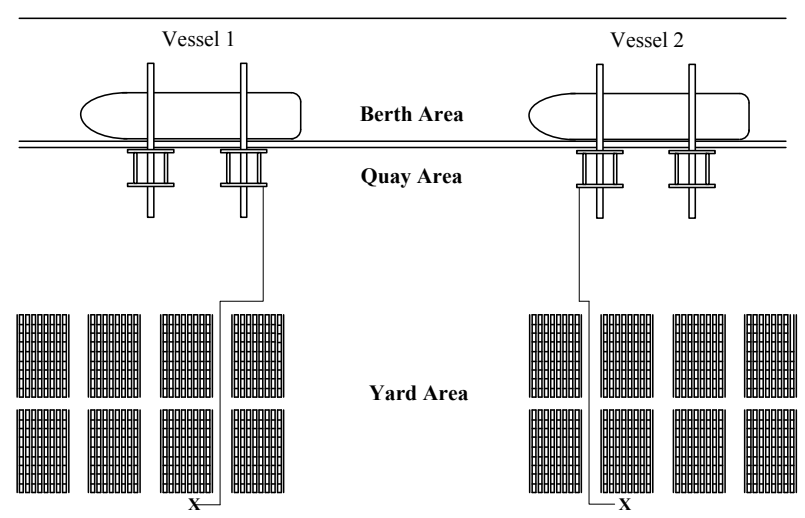

a) containers are discharged to their final position

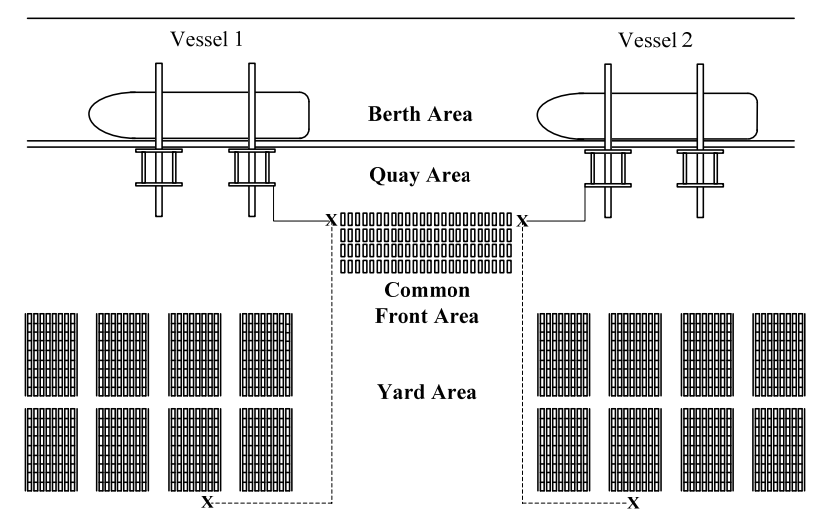

b) containers are discharged to a buffer area and transferred afterwards

Figure 3: Direct container discharge vs indirect container discharge in a front buffer area

The buffer area may also be conceived as individual container slots within the normal yard blocks of the terminal, yet, again, with a temporary purpose, as depicted by the example in Figure 4. Here the containers discharged from vessel 1 are first stacked within one yard block and later moved to another block from which they will be fetched for boarding on vessel 2 .

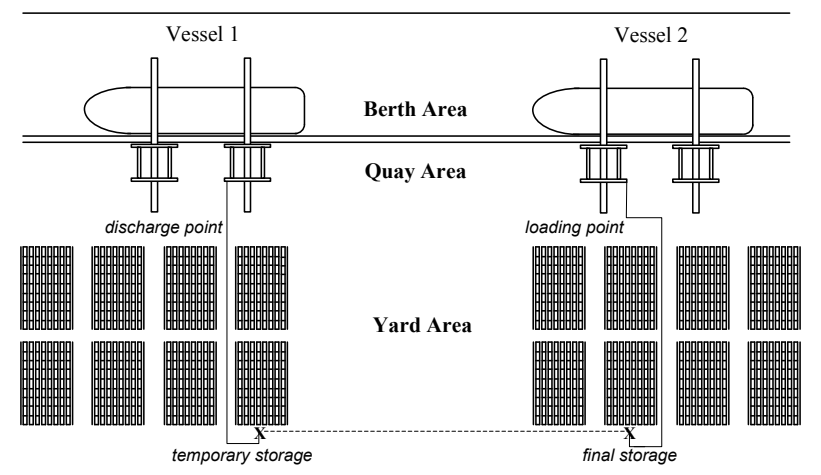

Figure 4: Indirect container discharge using temporary storage positions

With respect to the possible options for off-line planning, container discharge and loading operations have been discussed in a separate way. However, they are logically performed in sequence. To fix ideas, one may refer to a maritime container terminal for pure transshipment in which containers are transferred for further transportation from one vessel to another. As previously stated, during discharge operations containers are often left in temporary yard positions to speed up operations. On the other hand, the same containers are often then moved closer to the vessel boarding area in order to speed up operations by undergoing re-marshalling activities or preparing for container loading. Thus, discharge and loading operations are actually coupled by internal transfer operations also called housekeeping.

Housekeeping operations contribute to minimizing the vessel turn-around time by transferring containers from a temporary yard position or source block to a final position or destination block which is usually at a close range from the vessel boarding area (see Figure 4). These operations can be carried out by combining different types of transportation vehicles (e.g. straddle carriers, multi-trailers, reach stackers, AGVs), according to terminal infrastructures, the distance to be covered or the type of container to undergo transfer. Whatever be the case, container housekeeping involves the same organizational issues, as well as availability of human and mechanical resources required to perform "regular" container transfer 
between the yard and quay areas. As a matter of fact, not only are these operations held on the same grounds and share the same resources, but they also may interfere one with the other.

Unlike re-marshalling operations, to our knowledge, very little research has been done to address housekeeping with respect to performance evaluation under resource sharing in such complex and dynamics systems. A few studies have considered this topic, but only as a conceptual choice lying behind yard operating rules at the basis of empirical considerations and investigations for intelligent yard stacking (Chen 1999; Saanen and Dekker 2007a; Saanen and Dekker 2007b). In the present paper we will model housekeeping operations within maritime container terminals that are considered pure transshipment hubs and bear extensive storage yards. A discrete-event simulator will be used to $i$ ) assess the efficiency of the housekeeping operations under unforeseen events or process disturbances and ii) estimate the related productivity and waiting phenomena which, in turn, affect the vessel turn-around time. The rest of the paper is organized as follows. The simulation model of the housekeeping operations is described in detail in Section 2. Numerical experiments for scenario analysis via a what-if approach are reported in Section 3. Finally, conclusions are drawn in Section 4.

\section{HOUSEKEEPING MODEL}

\subsection{Conceptual Model}

The conceptual model of the housekeeping operations was tailored to the container terminal at the Port of Gioia Tauro in Southern Italy, however it is easily adaptable to suit any other type or size of terminal layout. The model provides a detailed representation of a berth sub-system, which is variable in length and adjacent to the yard sub-system. The yard is organized in blocks that, in turn, are grouped into four areas. Area $\mathrm{A}$ is located in front of the berth and most of it is devoted to handling 1-TEU operating (i.e. nonempty) containers; area B is immediately behind and used to handle 2-TEU operating containers; area C contains refrigerated (a.k.a. reefer) operating containers of both sizes; at last, all the non-operating containers are assigned to area D, the most distant from the berth. Every single area is organized in rows and every row is divided into equally distanced 1-TEU container slots. Blocks also feature multiple tiers for container stacking. The number of rows, slots and tiers depends on the various areas.

The model focuses on container housekeeping activities occurring between yard blocks, while it does not provide a detailed representation of the container discharge/loading operations performed on the berthed vessels. However, container transfer from and to the vessels in the berth sub-system is equally represented: these "regular" container flows are generated according to the various vessel services assigned to the single berth areas. For both housekeeping and regular traffic, container transfer and stacking/retrieval is carried out by straddle carriers (SCs).

\subsection{Simulation Model}

To model housekeeping operations we decided to use a queuing-based network whose representational capabilities have been enriched with additional graphical elements. In particular, fork and join actions on entities, as well as seize and release actions on resources have been included (see Figure 5).

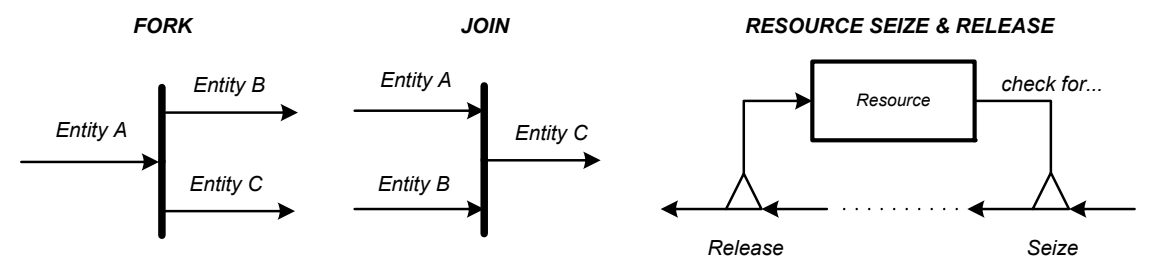

Figure 5: The additional graphical elements introduced in the housekeeping queuing model 


\section{Legato, Mazza, and Trunfio}

Fork and join operators are required to explicitly represent the life-cycle of the entities flowing through the model, that is containers when these are transferred from a yard slot to another (i.e. source block - destination block) and SCs when these are assigned to containers for their transfer.

Seize and release operators allow to manage the request of (a limited number of) resources on behalf of model entities. In particular, once an SC accesses a row of a yard block to perform container handling operations, the operator locks out the row at hand, along with the two adjacent rows, in order to avoid interference with other vehicles. Therefore it practically implements a non-simultaneity constraint on the container pick-up/set-down operations on a specific row of the yard block. In this sense, an example is given by the block in Figure 5: the "seize" triangle works like a semaphore by verifying if the resource (or part of it) can be accessed by the entity; after the seize block assigns the resource requested by the entity and operations are completed, the same entity will free the resource it holds by flowing through the "release" triangle.

The queuing model of the housekeeping process is illustrated in Figure 6 (the yard blocks in which housekeeping occurs are represented as rows enclosed by an outer border). The model is implemented according to the holistic approach by (Pidd and Castro 1998) which, in our opinion, better supports the representation of such a complex system (see Legato, Gullì, and Trunfio 2008). In this approach, one or more "manager" objects supervise complex dynamics like, for example, resource or task allocation at runtime.

The core of the model consists in the manager object called "SC Dispatcher". This object accounts for two functions: $i$ ) the identification of the containers to be housekept and $i$ ) the routing of the SCs towards the yard blocks in which container retrieval and delivery for both housekeeping and "regular" operations must be performed.

The first function identifies the type of container according to the occupancy level of the yard blocks and the fraction of containers usually housekept in each of the four yard areas (both represented by probability laws inferred from the statistical analysis of historical data).

The second function provides for the routing of SCs involved in housekeeping, as well as SCs engaged in "regular" container discharge/loading on the quay area. In the first case, the source/destination blocks are identified according to the list of containers to be housekept in the time period under examination. In the second case, requests for SCs to perform container discharge/loading operations on the quay area are generated by the so-called "Quay side" block. This object generates flows of SCs to/from the various blocks on the yard that are consistent with the vessel services assigned to the yard blocks. SCs are then routed by the SC Dispatcher towards the proper yard blocks according to the type of container to be delivered/retrieved or the type of movement to be performed (loading/discharge).

For greater comprehension, a step-by-step description of the sequence of housekeeping activities portrayed by the model in Figure 6 follows. When an empty $S C$ is available, the SC Dispatcher assigns a "housekeeping container" to the SC according to the actual position of the vehicle. As a result of the assignment, the SC holds all the necessary information for container transfer (row, slot and tier in the source block and designation of the destination block). Obviously, if multiple SCs are available, the SC Dispatcher assigns the SC that is closest to the location of the target container. Observe that the assignments performed by the SC Dispatcher are based on a greedy-type allocation policy which may lead to possible congestions in the source and destination blocks.

The SC then moves from its current position to the base of the row in the source block. The related travel time is generated by considering $i$ ) the distance between the last known position of the SC and the base of the yard block and $i$ ) a variate of the random variable "vehicle speed".

Once in front of the row in which the container to be housekept is located, the SC joins the corresponding $S C$ waiting line and (eventually) waits for its turn to access the row. The waiting time is due to the impossibility of carrying out operations simultaneously in the same row of a yard block. In fact, access operations to the row are filtered by seize and release operators on the rows of the block. Therefore, the SC can gain access to the container only when the row hosting the container and the two adjacent rows are not involved in handling operations (obtain rows). Obviously, loading operations start when both an SC and the container box to be retrieved are available. The loading time is given by the sum of 
the time required to move from the waiting position to the container slot, the pick-up time and the eventual shifting time. The latter occurs every time a container requiring transfer is stacked underneath one or more containers and it depends on the type of block (for instance, blocks in the D Area bear a greater number of tiers and, thus, shifting time for containers in this area is usually greater).

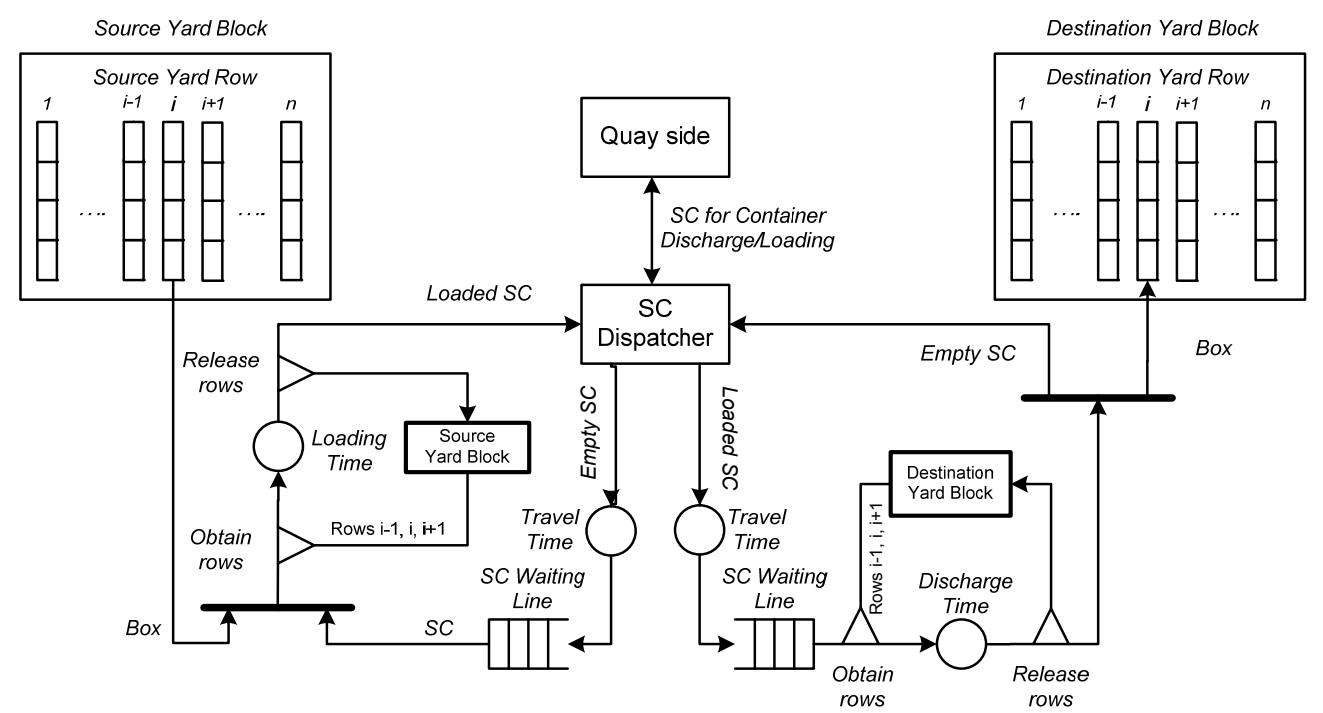

Figure 6: The housekeeping model

As soon as container loading operations finish, the SC releases the previously acquired rows (release rows) and returns loaded to the SC Dispatcher. It then queries the dispatcher with respect to the row and slot of destination (while the destination block is determined previously). As a consequence, the identified row and slot are reserved for the arriving container.

The sequence of operations for container delivery is analogous to the one for container retrieval. In brief, the loaded SC moves from the slot in the source block to the queue before the row in the destination block. The procedure for row access is repeated and, once the container discharge operation is finished, the SC queries the SC Dispatcher with respect to its new destination.

For sake of completion, observe that if the SC is dedicated to regular container discharge/loading operations on the quay instead of housekeeping, in the same way the SC Dispatcher routes the SC to/from the quay to carry out container delivery/retrieval operations.

\section{NUMERICAL EXPERIMENTS}

In this section the current practice for housekeeping operations in the container terminal in Gioia Tauro is compared to alternative housekeeping settings and policies. In particular, comparison is addressed by performing what-if analyses via discrete-event simulation and considering different settings (what-if experiments $\mathrm{n}^{\circ}$, i.e. the number of SCs to be deployed in housekeeping operations), policies (what-if experiments $\mathrm{n}^{\circ} 2$, i.e. single stacking vs multiple stacking in the yard blocks), and events (what-if experiments $\mathrm{n}^{\circ}$ 3, i.e. increase of container traffic due to non scheduled vessel arrivals or traffic reassignment). The objective of the what-if analyses is to $i$ ) assess the efficiency of the current housekeeping operations under unforeseen events or process disturbances and $i$ ) estimate the related productivity and waiting times which, in turn, affect the vessel turn-around time.

The design and implementation of the simulator depicted in Figure 7 has been carried-out in compliance with all the conventional steps used to guide a thorough and sound simulation study (Banks et al. 2001). However, due to the role of input data as driving force for the simulation model and the im- 
portance of having confidence in the "predictive" capability of the simulation model, we explicitly report on the input modeling, verification and validation steps of the study.

Input data for the problem at hand has been collected and analyzed for both describing system features (e.g. area sizes or equipment number and speed) and performing system analysis.

For the first category, the main features of the housekeeping process referring to the yard and handling equipment have been explicitly modeled according to the current terminal organization and practice. In particular, the yard has been divided into 4 major areas, $\mathrm{A}, \mathrm{B}, \mathrm{C}$ and $\mathrm{D}$, each of which is characterized by 18 blocks. In turn, each block has (on average) 16 bays, 32 rows and 3 tiers. The initial occupation level of the yard, i.e. the percentage of slots already occupied by containers, has been generated to mirror the actual state of the terminal yard at a given moment in time. Observe that this initial configuration, which has been set at $60 \%$, is then dynamically modified by container transfer due to housekeeping, as well as regular traffic occurring between the quay area and the yard. The latter of these flows also feeds the housekeeping process if the distance to be covered by the SCs providing for container stacking on the yard is greater than a threshold value which in our case has been set to $700 \mathrm{~m}$. As for the handling equipment, 10 SCs have been dedicated to housekeeping operations and they travel on the yard at a speed of 20 $\mathrm{km} / \mathrm{h}$ with containers and $30 \mathrm{~km} / \mathrm{h}$ without containers.

In terms of input data for system analysis, by means of statistical inference on the terminal's historical data, different types of distribution probability functions have been identified as "candidate" representations of the theoretical data models of the operation durations that bear the major sources of uncertainty:

- regular traffic of containers related to different shipping lines, but whose interarrival times are all well captured by density functions of the gamma family (the cumulative traffic rate is equal to 125 containers $/ h$ );

- container shifting time whose profile is well fitted by a triangular density function (mode $=1 \mathrm{~min}$, $\min =0.5 \min , \max =4 \min$;

- container pick-up/set-down time whose profile agrees with an exponential density function (mean=1min).

As far as verification is concerned, in order to verify if the input parameters and logical structure of the conceptual model are correctly represented by the simulation model, three classes of techniques have been used: common-sense techniques, thorough documentation and traces.

During validation, to assess if the real system is accurately represented by the simulation model, besides discussing the structural assumptions and data assumptions with the key figures of the real system, model input-output transformations have been compared to the corresponding input-output transformations for the real system. As a result of both of these validation activities, one can appreciate the capability of the simulator to mirror the real system performance by observing the figures in Table 1 .

Table 1: Real housekeeping performance measures referred to SCs are compared with the corresponding simulation results for validation purposes

\begin{tabular}{|l|c|c|}
\hline \multirow{2}{*}{ Source } & \multicolumn{2}{|c|}{ SCs } \\
\cline { 2 - 3 } & $\begin{array}{c}\text { Throughput } \\
{[\text { containers/SC } \cdot \mathrm{h}]}\end{array}$ & $\begin{array}{c}\text { Distance covered } \\
{[\mathrm{km} \text { per } \mathrm{h}]}\end{array}$ \\
\hline Company records & 5 & 16.88 \\
\hline Simulator & {$[4.97-5.03]$} & {$[16.65-17.10]$} \\
\hline
\end{tabular}

In terms of general settings, performance measures are estimated by simulating 150 runs of each given scenario in a 6-hour time horizon (i.e. one shift), hence, the simulator can provide both point and interval estimates. As for common scenario settings, a 3000-meter yard and a workload of nearly 500 housekeeping containers are considered across the experiments.

The first what-if analysis consists in investigating how the performance measures of the housekeeping process vary by changing the number of SCs dedicated to the housekeeping operations. In terms of pro- 
cess disturbance recalled by objective $i$ ), this allows to account for the effect of the starvation or congestion phenomena that eventually arise if the number of the SCs is not appropriate for the current housekeeping scenario. Let $n$ be the number of SCs. Experiments are carried out by bearing in mind that the company currently sets $n=10$ to handle the housekeeping containers.

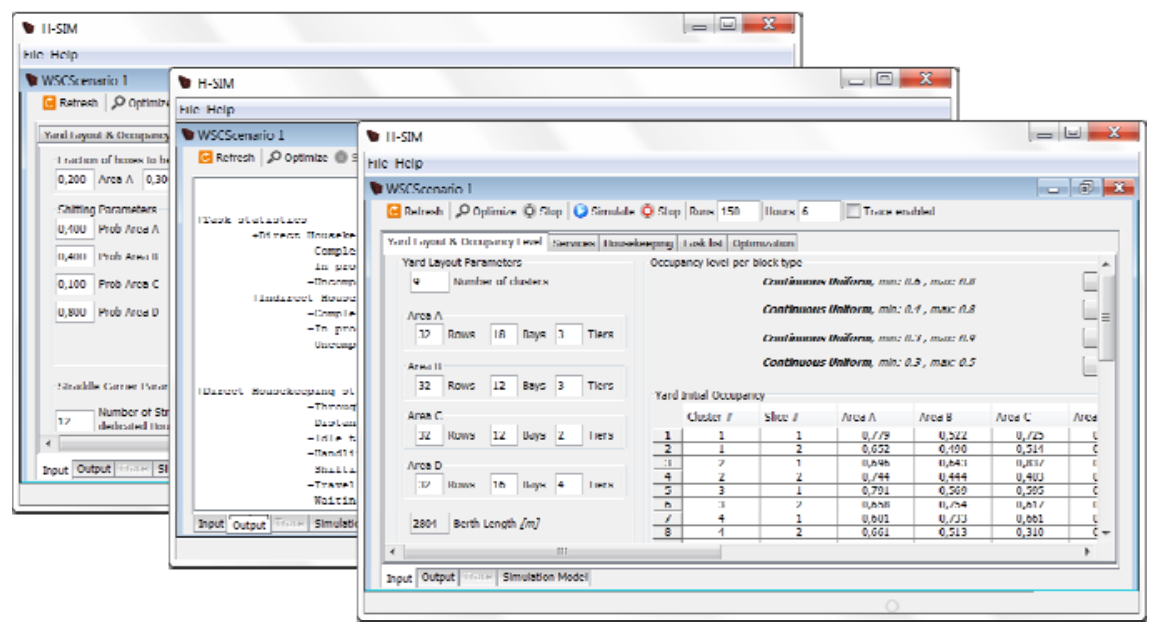

Figure 7: Some snapshots of the housekeeping simulator

The two charts illustrated in Figure 8 compare the trends of two correlated housekeeping performance measures: completion level and SC throughput. The former measures the progress made in the number of containers to be housekept during the shift (out of the 500 planned). The latter measures the number of containers housekept by each SC per hour.

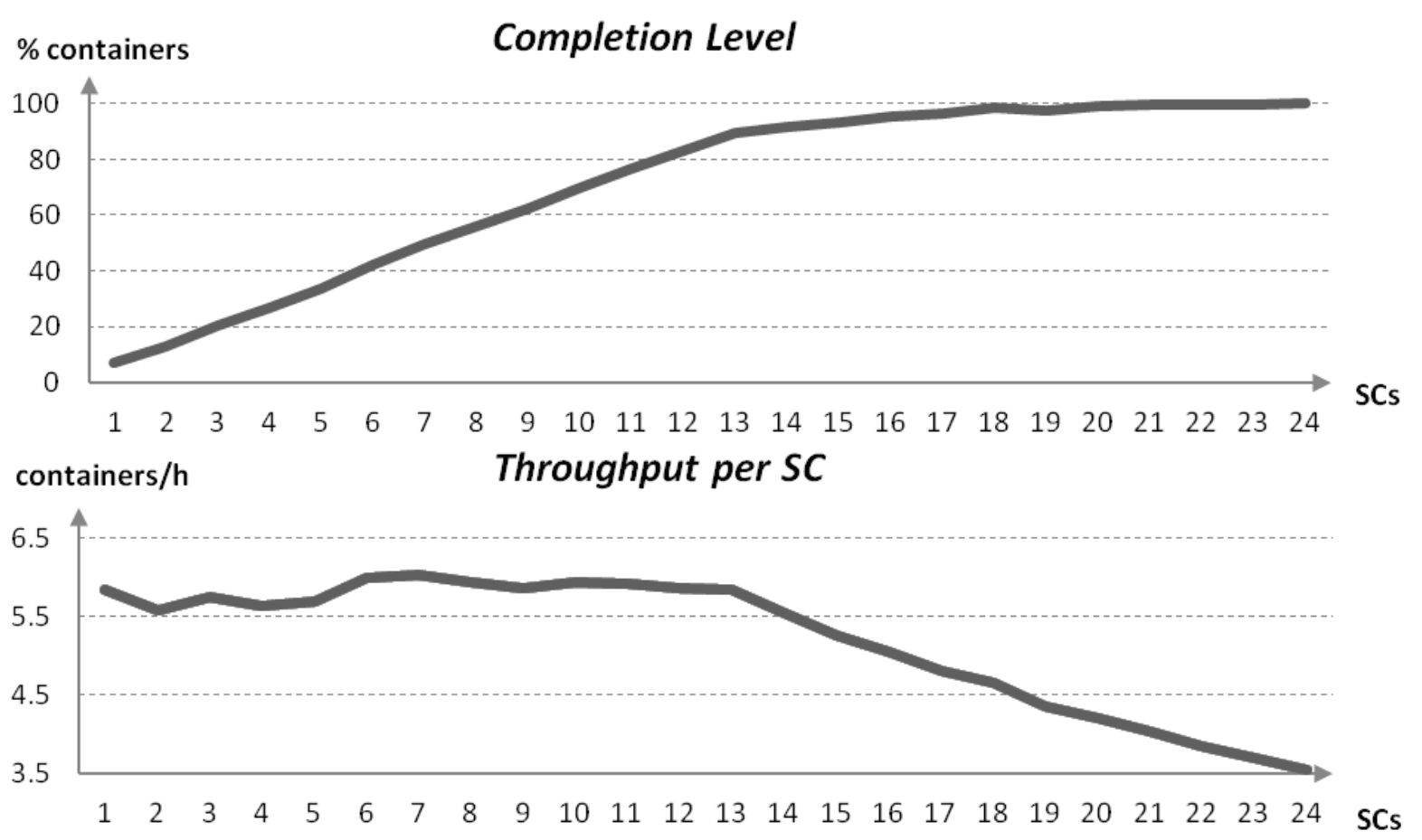

Figure 8: The overall number of containers handled and the hourly throughput per SC estimated by varying the number of SCs used for the housekeeping process 
Looking at Figure 8, one may observe that the number of containers cumulatively handled by all the SCs increases linearly with $n$ up to an elbow value, while the throughput per SC remains constant with $n$. Beyond the $n$ value corresponding to the elbow peak, as one may expect, the throughput remains constant and the productivity per SC decreases. For the case at hand, rather than keeping the current practice at $n=10$, the operations manager may push it up to $n=13$. Beyond this value, the marginal utility of allocating additional SCs to the housekeeping process in the given work shift goes to zero.

The second what-if analysis is carried out by considering the opportunity of varying the number of tiers to be enabled per yard block with respect to the current practice (i.e. 3 tiers for the blocks with operative containers). In terms of process disturbance recalled by objective $i$ ), this allows to account for the effect of the congestion phenomenon that eventually arises from an oversized tier height and, thus, the inflated operation time required by SCs to perform container shifting for those units that are not located on the top of the tiers in the current housekeeping scenario. Let $t$ be the number of tiers. In principle, $t$ could be fixed at will in order to increase the yard capacity of the container terminal; in practice, $t$ is constrained by the physical limits of the mechanical resources adopted for container stacking and retrieval (e.g. gantry cranes, SCs, reach stackers).

Five different scenarios on the current yard layout are tested by varying $t$ from 1 to 5 . When $t=1$, no shifting time for the container handling is required, since all the containers are on the ground. Vice versa, as $t$ increases, a target container could be underneath other containers and, therefore, an increasing handling time is expected as well.
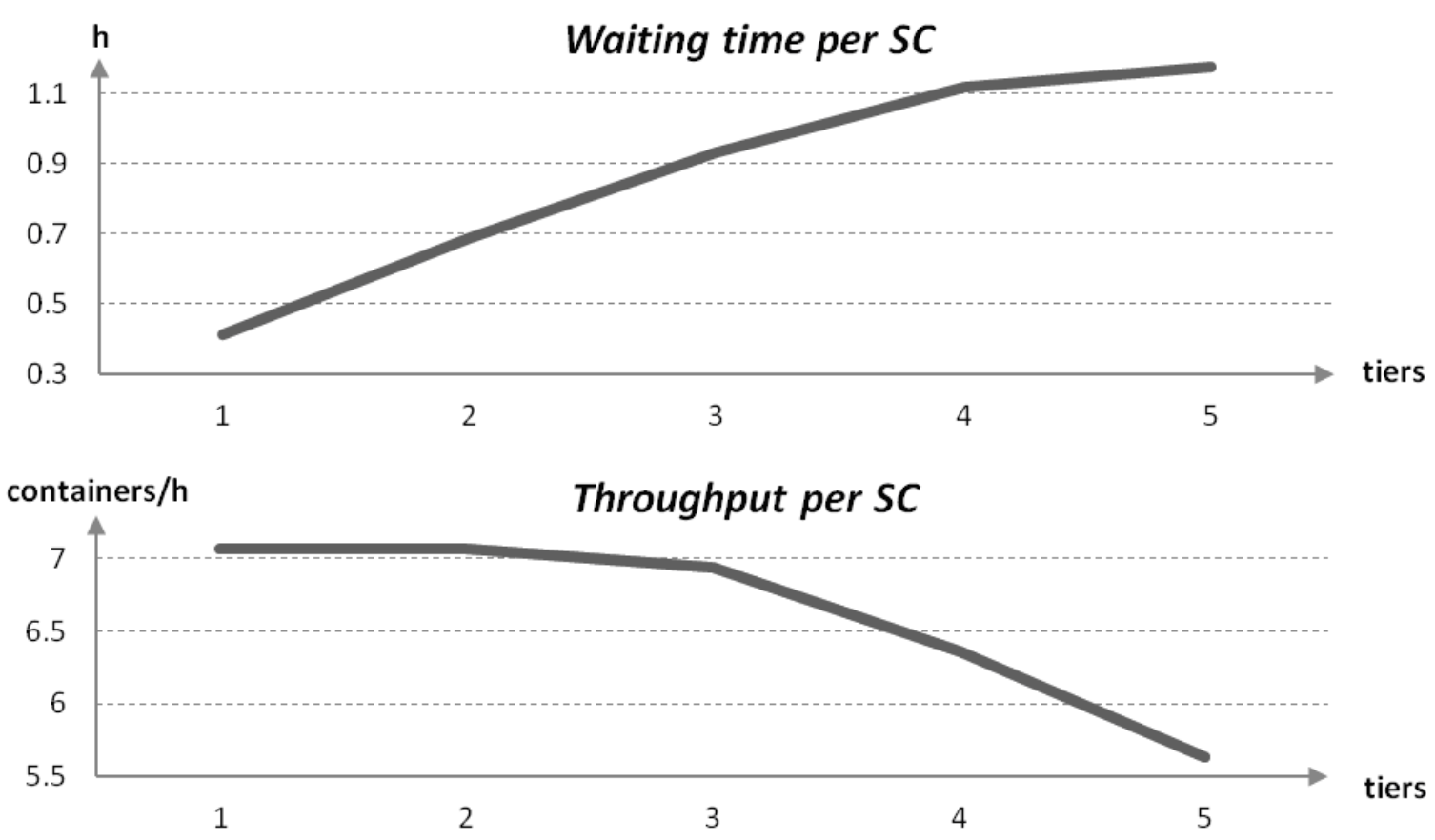

Figure 9: The hourly throughput and the total waiting time per SC estimated by changing the number of tiers of the yard blocks

Figure 9 reports the performance results of the scenario analysis described in terms of waiting time and throughput per SC. While the latter has been previously defined, the former measures the time spent by an SC waiting to access a specific row in a yard block (see Figure 6) and, as expected, it grows with the number of tiers. Numerical experiments support the adequacy of the current terminal practice and recommend to avoid $t>3$. In particular, one may observe that for $t=3$ the waiting time increases by ten minutes per hour, but the yard capacity triplicates without loss on SC throughput. 
In the final set of experiments, the impact of a growing "regular" container traffic flow on performance measures of the housekeeping operations is estimated. In terms of throughput and waiting time per SC recalled by objective ii), this allows to account for the effect of eventual "spot arrivals" (i.e. non scheduled vessel arrivals) or a proposal/need of change in vessel service-space allocation on the yard (e.g. maintenance to be carried out on a given yard area could cause the related portion of regular traffic to be concentrated in another area) in the current scenario. Specifically, for illustrative purposes, the growing weight of the regular SC traffic between the quay and yard areas is simply captured by the probability that a housekeeping SC is prevented to access a yard row due to the presence of other vehicles. The related performance profiles are plotted in Figure 10.
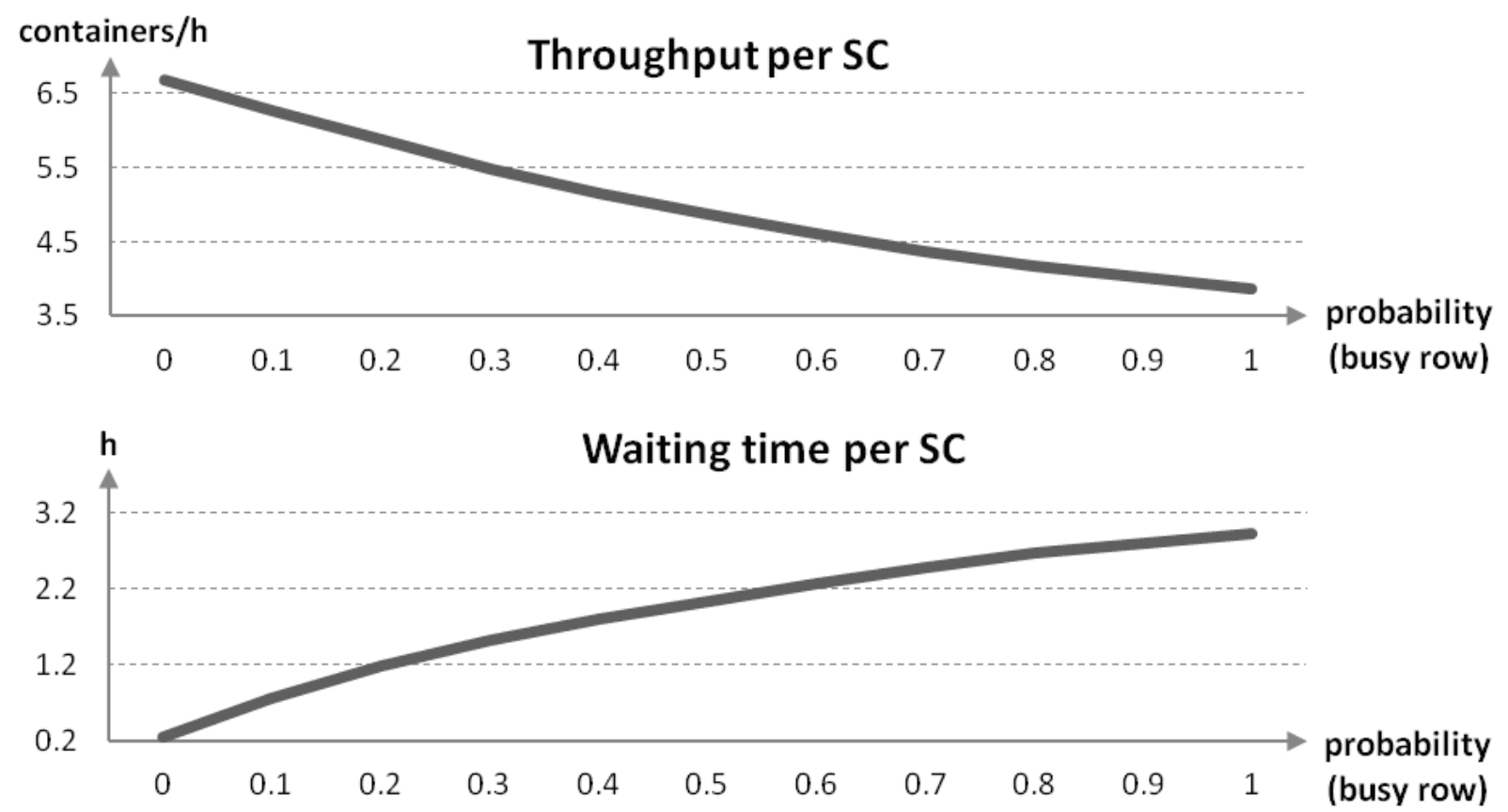

Figure 10: The hourly throughput and the total waiting time per SC estimated for increasing values of the probability of finding the row of a yard block busy

Clearly, when $p=0$ (i.e. there are no regular discharge/loading operations being performed on the quay during the time-horizon), there is likely to be no congestion on the yard blocks; the only waiting time due to housekeeping is small and the throughput per SC is maximized. Approaching $p=1$ (i.e. interference with regular SC traffic on the yard rows occurs continuously during the work shift), the inflating waiting time causes a drop in the throughput of the housekeeping SCs. In this sense, the operations manager could fix a target value for both performance measures in order to better support the eventual decisions on regular traffic reallocation.

\section{CONCLUSIONS AND FUTURE WORK}

A queuing-based network model has been proposed to represent the housekeeping process in a maritime container terminal. The performance of the handling equipment and the current housekeeping practices deployed in a real facility have been evaluated by a discrete-event simulator. According to the numerical experiments presented, in the current terminal configuration: $i$ ) as many as 13 SCs can be dedicated to housekeeping operations in order to increase the number of containers cumulatively handled, without loss of throughput per SC; ii) tier height equal to 3 provides the best compromise between SC waiting times and yard capacity, without loss on SC throughput in housekeeping; iii) the operations manager needs to 
fix a target value for SC waiting times and throughput in housekeeping operations when deciding on regular traffic reallocation.

The above simulator has also been conceived to be the kernel of a soon-to-be simulation-based optimization (SO) framework, by which current practices may be replaced by optimized policies and tested on a real-time basis. According to the logic of this framework, the initial assignment and order, or schedule, of the containers to be housekept by dedicated SCs can be provided by the terminal practice. Then simulation and optimization modules are iteratively combined to obtain the best schedule with respect to the performance measures of interest. A metaheuristic based algorithm is under development to explore the set of feasible schedules, which are evaluated via simulation and then compared, by balancing intensification and diversification efforts within the search process that drives the global SO procedure.

\section{REFERENCES}

Banks, J., J. S. Carson, B. L. Nelson, and D. M. Nicol. 2000. Discrete-Event System Simulation. 3rd ed. Upper Saddle River, New Jersey: Prentice-Hall, Inc.

Bortfeldt, A. and F. Forster. 2012, "A Tree Search Procedure for the Container Pre-marshalling Problem." European Journal of Operational Research 217:531-540.

Chen, T. 1999. "Yard Operations in the Container Terminal - A Study in the 'Unproductive Moves'." Maritime Policy \& Management: The Flagship Journal of International Shipping and Port Research 26(1):27-38.

Choon, T. K., L. P. Ku, L. H. Lee, and E. P. Chew. 2010. “An Optimisation Framework for Yard Planning in a Container Terminal - Case with Automated Rail-Mounted Gantry Cranes." OR Spectrum 32:519-541.

Container Trades Statistics. "Monthly e-newsletters with Total Trade Volumes for Dry and Reefer Containers and Aggregated Price Indices for Seven Trades to and From Europe." Accessed March 26, 2012. http://www.containerstatistics.com/newsletter.

Hirashima, Y. 2009. “A Q-learning System for Container Marshalling with Group-Based Learning Model at Container Yard Terminals." In Proceedings of the International MultiConference of Engineers and Computer Scientists 2009 (IMECS 2009) Volume I, March 18-20, 2009, Hong Kong.

Huang, S.-H. and T.-H. Lin. 2012. "Heuristic Algorithms for Container Pre-marshalling Problems." Computers \& Industrial Engineering 62:13-20.

Kim, K. H. and J. W. Bae. 1998. "Marshaling Export Containers in Port Container Terminals." Computers and Industrial Engineering 35(3):655-658.

Lee, Y. and S.-L. Chao. 2009, "A Neighborhood Search Heuristic for Pre-marshalling Export Containers." European Journal of Operational Research 196:468-475.

Lee, Y. and N.-Y. Hsu. 2007. "An Optimization Model for the Container Pre-marshalling Problem." Computers \& Operations Research 34:3295-3313.

Legato, P., D. Gullì, and R. Trunfio. 2008. "Modelling, Simulation and Optimization of Logistic Systems." In Proceedings of the 20th European Modeling and Simulation Symposium (Simulation in Industry), 569-578, Amantea (Italy).

Pidd, M. and R. B. Castro. 1998. "Hierarchical Modular Modelling in Discrete Simulation." In Proceedings of the 1998 Winter Simulation Conference, Edited by D.J. Medeiros, E.F. Watson, J.S. Carson, and M.S. Manivannan, 383-389, Washington DC, USA. Piscataway, New Jersey: Institute of Electrical and Electronics Engineers, Inc.

Saanen, Y. A. and R. Dekker. 2007a. "Intelligent Stacking as Way Out of Congested Yards? Part 1." Port Technology International 31:87-92.

Saanen, Y. A. and R. Dekker. 2007b. "Intelligent Stacking as Way Out of Congested Yards? Part 2." Port Technology International 32:80-85. 
Yu, V. F., H.-Y. Cheng, and H.-I Ting. 2009. "Optimizing Re-marshalling Operation in Export Container Terminals." In Proceedings of the $10^{\text {th }}$ Asia Pacific Industrial Engineering \& Management System Conference (APIEMS 2009), December 14-16, 2009, Kitakyushu, Japan.

\section{AUTHOR BIOGRAPHIES}

PASQUALE LEGATO is an Associate Professor of Operations Research at the Faculty of Engineering University of Calabria, Rende (CS, Italy), where he teaches courses on simulation for system performance evaluation. He has published on queuing network models for job shop and logistic systems, as well as on integer programming models. He has been involved in several national and international applied research projects and is serving as a reviewer for many international journals. His current research activities focus on the development and analysis of queuing network models for logistic systems, discrete-event simulation and the integration of simulation output analysis techniques with combinatorial optimization algorithms for real-life applications in Transportation and Logistics. His home-page is www.deis.unical.it/legato.

RINA MARY MAZZA received her Laurea degree in Management Engineering and obtained a Ph.D. degree in Operations Research at the University of Calabria, Rende (CS, Italy). She is currently head of the "Research Projects Office" at the Department of Electronics, Computer and System Sciences (DEIS) of the above University. She has a seven-year working experience on Knowledge Management and Quality Assurance in Research Centers. She is also a consultant for operations modeling and simulation in terminal containers. Her current research interests include discrete-event simulation and optimum-seeking by simulation in complex logistic systems. Her e-mail address is rmazza@deis.unical.it.

ROBERTO TRUNFIO received his M.Sc. in Management Engineering (cum laude) from the University of Calabria, Rende (CS, Italy) in 2005. In March 2009, he received a Ph.D. in Operations Research from the Department of Electronics, Computers and Systems (DEIS) at the same university, focusing his research on modeling, simulation and optimization in logistics. Currently, his research activity lies in simulation, optimization and simulation-based optimization, with emphasis on container terminal logistics. His email address is rtrunfio@deis.unical.it and his web page is http://www.trunfio.it. 